\title{
Spatio-temporal variability of bovine tuberculosis eradication in Spain
}

\section{(2006-2011)}

Garcia-Saenz A ${ }^{1 *}$, Saez $M^{2}$., Napp $S^{1}$., Casal $\mathrm{J}^{1,3}$., Saez $\mathrm{J}^{4}$., Acevedo $\mathrm{P}^{1,5}$., Guta $\mathrm{S}^{1,6}$, Allepuz $\mathrm{A}^{1,3}$.

(1) Centre de Recerca en Sanitat Animal (CReSA), UAB-IRTA, Campus de la Universitat Autònoma de Barcelona, 08193 Bellaterra, Barcelona, Spain

(2) Research Group on Statistics, Applied Economics and Health (GRECS), University of Girona, 17004 Girona, Spain

(3) Departament de Sanitat i Anatomia Animals, Universitat Autònoma de Barcelona, 08193 Bellaterra, Barcelona, Spain

(4) Subdirección General de Sanidad de la Producción Primaria, Dirección General de Recursos Agrícolas y Ganaderos, Ministerio de Medio Ambiente, y Medio Rural y Marino, 28071 Madrid, Spain

(5) CIBIO, Centro de Investigacao em Biodiversidade e Recursos Geneticos, Universidade do Porto Campus Agrario de Vairao, 4485-661 Vairao, Portugal

(6) National animal health diagnostic and investigation center (NAHDIC), P.O. Box 04, Sebeta, Ethiopia

*Corresponding author. Tel.: +34 93581047; fax: +34 935812006.

E-mail address: ariadna.garcia@cresa.uab.cat 


\section{Summary}

In this study we analyzed the space-time variation of the risk of bovine tuberculosis (bTB) in cattle between 2006 and 2011, and identified factors related with the variability of the risk of infection.

The results indicated that at country level, there was no significant temporal trend between consecutive years in the risk of being positive or newly positive. However, at county level bTB evolution was more heterogeneous, and some counties presented in a specific year a significant increase or decrease in the risk of being infected or newly infected indicating, and it is related with the presence of short-latency underlying factors.

The analysis of potential risk factors indicated that both, a large number of movements from counties with high incidence (>1\%) and presence of bullfighting cattle herds, had a positive relation with the risks of a county being positive or new positive. However, these results should be interpreted with care, and the correlations found at aggregated-level need to be confirmed at farm-level by other types of epidemiological studies.

\section{Keywords}

Bovine tuberculosis, Space-time analysis, Eradication program, Spain, R-INLA 


\section{Introduction}

Bovine tuberculosis (bTB) is a chronic infectious disease caused by Mycobacterium bovis, a gram positive bacillus that belongs to the $M$. tuberculosis complex (MTBC) (de la Rua-Domenech et al., 2006). The disease can affect a wide range of domestic and wild animals (Phillips et al., 2003; Humblet et al., 2009; Gortázar et al., 2012), and due to its zoonotic nature and the high economic impact in livestock production, most of the developed countries where the disease is endemic have established bTB eradication programs (Reviriego Gordejo \& Vermeersch, 2006).

In Spain, the first bTB testing was performed in 1950 in a dairy herd in the north of Spain, but it was not until 1993 when all dairy and beef herds were included in the control program. During the application of this program, mainly based on intradermal tuberculin testing (IDT) and culling of reactor animals, the cattle herd prevalence has decreased from $5.9 \%$ in 1993 to $1.3 \%$ by the end of 2011 (Anon, 2012a). Despite that important progress, during the last 12 years, the herd prevalence in Spain has only declined from $2.5 \%$ in 2000 to $1.3 \%$ in 2011 , and the herd incidence has been fluctuating between 0.8 and $1.0 \%$.

Spatio-temporal disease mapping models are a useful tool to describe the pattern of diseases and to identify regions with unusual levels of disease, time trends or both (Meliker et al., 2011; Schrödle and Held, 2009). Moreover, space-time models can contribute to the assessment of the stability of the estimated spatial patterns of disease, which cannot be evaluated just by spatial models (Abellan et al., 2008). Following Lawson et al. (2009), when analyzing the observed counts of disease within different areas for a sequence of time periods (i.e. space-time analysis), three groups of components can be considered: i) the spatial dimension: area-specific risk factors such as contact with infected wildlife or characteristics of the farms within the county (e.g. 
herd size or livestock rearing practices), ii) the space-time dimension: time-area specific risk factors, such as changes between years in the county due to new personnel, diagnostic procedures or movements of animals, and finally, iii) the temporal dimension: time risk factors such as global changes in the national eradication program. Moreover, the decomposition of the risk variability by using different (and appropriate) random effects allows the formulation of hypotheses about the role of factors potentially related to the risk of infection. The different levels of variability in the observed cases of a disease can be accounted for by the use of multilevel (i.e., hierarchical) models (Beale et al., 2008).

In a previous analysis about the geographical risk of bTB infection across Spain, the spatial bTB risk heterogeneity across the country was quantified and it was evidenced that counties located in the central and south of Spain had a risk more than three times bigger than the rest of the country (Allepuz et al., 2011). However, in that study the temporal evolution of the disease across areas was not assessed. Furthermore, specific covariates were not included in the model so their relationship with the risk of bTB could not be assessed.

The objective of the present work was to analyze the space-time variation in the risk of bTB in cattle between 2006 and 2011, and to identify factors related with the variability of the risk of infection.

\section{Materials and Methods}

\section{Data collection and management}

For the period 2006-2011, annual data on the total number of herds, bTB positive herds and new positive herds (i.e., herds that became positive within a given year) at county level were provided by the Spanish Ministry of Agriculture, Food and Environment (MAGRAMA). The information about some explanatory covariates 
included in the model: number of herds in the county by type (dairy, beef or fighting bulls), number of cattle-goat mixed herds, number of goat farms and animal movements between counties from 2006 to 2011, was also provided by MAGRAMA.

The abundance of red deer in Spain was obtained at UTM 10x10 $\mathrm{km}^{2}$ grid cells from Acevedo et al. (2010). The abundance of red deer was aggregated at county level by calculating the average abundance of the different cells that intersected with the county. This data aggregation was carried out through Quantum GIS vs. 1.8.0 by joining the attributes based on their spatial location.

For each year, three variables related to animal movement were created: i) in-degree, i.e., the number of counties from which a given county received animals, which was calculated using the statnet package (Handcock et al., 2003); ii) number of animal movements to a given county (including intra-county movements), and iii) the number of animals moved to a given county (including animals from intra-county movements). Besides, a differentiation was made between high risk movements (i.e., from counties with an incidence $>1 \%$ ), and low risk movements (i.e., from counties with an incidence $\leq 1 \%$ ), resulting in six explanatory covariates. Figures 1 and 2 show the distribution of the categories for each of the variables included in the model.

All the explanatory variables were included in the model as categorical variables in order to avoid problems derived from non-linear relationships with the dependent variable, and the possible concurvity between explanatory covariates and random factors (ref Marc). For this purpose, the covariates were reclassified into four categories following the quartile distribution. In the case of red deer abundance and number of mixed cattle-goat farms, as their first quartile was zero, they were also divided into four categories, but the first one included all the zeroes, and the remaining values were 
divided into terciles. Moreover, as half of the values of bullfighting cattle were zeroes, this variable was included as dichotomous (absence and presence).

\section{Model specification}

We assumed that the total and the new bTB positive herds at county level followed a Poisson distribution centered on $\lambda_{i j}$ and $\gamma_{i j}$, respectively, being $i$ the county (i.e., $i=1$ to 483) and $j$ the year (i.e., $j=2006$ to 2011 ):

$$
\begin{aligned}
& \lambda_{i j}=P_{i j} x H_{i j} \\
& \gamma_{i j}=I_{i j} x H_{i j}
\end{aligned}
$$

Where, $P_{i j}$ and $I_{i j}$ are the prevalence and incidence respectively, and $H_{i j}$ the total number of herds, in each county and year.

Due to the hierarchical structure of the data (i.e., space, time and space-time), a generalized linear mixed model (GLMM) approach was implemented (Zuur et al. 2009).

Within the Poisson regression and GLMM context, $\hat{P}_{i j}$ and $\hat{I}_{i j}$ were parameterized as a function of random and fixed effects:

$$
\hat{P_{i j}} \text { or } \hat{I}_{i j}=\exp \left(\beta_{0}+S_{i}+e t a_{i}+g_{j}+p s i_{i, j}+Z \beta+\epsilon\right)
$$

Where, $\beta_{0}$ represents the intercept, $S_{i}$ is the structured spatial random effect for the spatial dependence between counties, which was defined by a stochastic partial differential equation (SPDE), and $e t a_{i}$ is the unstructured spatial component. The temporal component $g_{j}$ and the space-time interaction $p s i_{i, j}$ random effects were defined by an autoregressive model of order 1 . Finally, $Z \beta$ represented the explanatory covariates, and $\in$ was the residual term (assumed to be approximately normally distributed).

The SPDE was calculated from a matrix of Euclidean distances between centroids of each county using Delaunay triangulation (Simpson et al., 2011; Cameletti et al., 2011). 
The spatial variation of the relative risk of the prevalence (RRP) and incidence (RRI) across the country was calculated dividing the estimated prevalence $\left(\hat{P}_{\mathrm{ij}}\right)$ or incidence $\left(I_{\mathrm{ij}}\right)$ in a given county and year, by the global prevalence or global incidence in that year, respectively.

The covariates were included into the model by the following procedure: in a first step, all possible combinations of the model with random effects and just one covariate were evaluated. The model with the lowest Deviance Information Criterion (DIC) value was selected. Then, all possible model combinations of the first selected model plus a new covariate were analyzed, and the model with the lowest DIC value was selected. These processes were repeated by adding new covariates until the final model, the model combination with the lowest DIC value, was selected (Held et al., 2010; Spiegelhalter et al., 2003).

To determine the statistical significance of the variables in the model, $95 \%$ credible intervals (CR) were obtained from the posterior probability distribution. If the $95 \% \mathrm{CR}$ was greater than 1 , the variable would be considered significantly positively correlated with the dependent variable. If the $95 \%$ CR was lower than 1, the variable would be considered significantly negatively correlated with the dependent variable.

All statistical analyses were performed using R software 2.14.1 (R Core Team 2012), and the models were fitted using the R-INLA package to avoid the algorithm problems of convergence and mixing of the Markov Chain Monte Carlo (MCMC)-based sampling methods (Bizancio et al., 2011; Schrödle and Held, 2010).

\section{Results}

1. Descriptive results 
The number of positive and new positive herds by year and type of herd are presented in Tables 1 and 2. For all the years of study, dairy cattle had the lowest percentage of positive and new positive herds, while fighting bulls had the highest proportion. Approximately $50 \%$ of the positive herds each year were new positives.

Table 1. Bovine tuberculosis (bTB) positive herds (Pos), number of herds (Herds) and percentage of bTB positive herds (\%), between 2006 and 2011.

\begin{tabular}{|c|c|c|c|c|c|c|c|c|c|c|c|c|}
\hline \multirow[t]{3}{*}{ Year } & \multicolumn{9}{|c|}{ Positive herds } & \multicolumn{3}{|l|}{ Total } \\
\hline & \multicolumn{3}{|c|}{ Beef } & \multicolumn{3}{|c|}{ Dairy } & \multicolumn{3}{|c|}{ Bullfighting } & \multirow[b]{2}{*}{ Pos } & \multirow[b]{2}{*}{ Herds } & \multirow[b]{2}{*}{$\%$} \\
\hline & Pos & Herds & $\%$ & Pos & Herds & $\%$ & Pos & Herds & $\%$ & & & \\
\hline 2006 & 2108 & 105,164 & 2.0 & 203 & 30,568 & 0.7 & 98 & 1184 & 8.3 & 2411 & 136,916 & 1.8 \\
\hline 2007 & 1809 & 99,236 & 1.8 & 199 & 29,012 & 0.7 & 120 & 1815 & 6.6 & 2128 & 130,063 & 1.6 \\
\hline 2008 & 1703 & 97,067 & 1.8 & 173 & 26,454 & 0.7 & 108 & 1220 & 8.9 & 1984 & 124,741 & 1.6 \\
\hline 2009 & 1683 & 92,684 & 1.8 & 159 & 25,767 & 0.6 & 128 & 1211 & 10.6 & 1971 & 119,662 & 1.6 \\
\hline 2010 & 1504 & 90,174 & 1.7 & 123 & 25,012 & 0.5 & 128 & 1209 & 10.6 & 1759 & 116,395 & 1.5 \\
\hline 2011 & 1304 & 86,083 & 1.5 & 97 & 24,219 & 0.4 & 84 & 1153 & 7.3 & 1488 & 111,455 & 1.3 \\
\hline
\end{tabular}

Table 2. Bovine tuberculosis (bTB) new positive herds (Pos), number of herds (Herds) and percentage of bTB new positive herds (\%), between 2006 and 2011.

\begin{tabular}{|c|c|c|c|c|c|c|c|c|c|c|c|c|}
\hline \multirow[t]{3}{*}{ Year } & \multicolumn{9}{|c|}{ New positive herds } & \multicolumn{3}{|c|}{ Total } \\
\hline & \multicolumn{3}{|c|}{ Beef } & \multicolumn{3}{|c|}{ Dairy } & \multicolumn{3}{|c|}{ Bullfighting } & \multirow[b]{2}{*}{ Pos } & \multirow[b]{2}{*}{ Herds } & \multirow[b]{2}{*}{$\%$} \\
\hline & Pos & Herds & $\%$ & Pos & Herds & $\%$ & Pos & Herds & $\%$ & & & \\
\hline 2006 & 987 & 105,164 & 0.9 & 136 & 30,568 & 0.4 & 43 & 1184 & 3.6 & 1166 & 136,916 & 0.9 \\
\hline 2007 & 1126 & 99,236 & 1.1 & 160 & 29,012 & 0.6 & 91 & 1815 & 5.0 & 1377 & 130,063 & 1.1 \\
\hline 2008 & 950 & 97,067 & 1.0 & 122 & 26,454 & 0.5 & 63 & 1220 & 5.2 & 1135 & 124,741 & 0.9 \\
\hline 2009 & 1039 & 92,684 & 1.1 & 119 & 25,767 & 0.5 & 73 & 1211 & 6.0 & 1231 & 119,662 & 1.0 \\
\hline 2010 & 837 & 90,174 & 0.9 & 96 & 25,012 & 0.4 & 57 & 1209 & 4.7 & 990 & 116,395 & 0.9 \\
\hline 2011 & 822 & 86,083 & 1.0 & 74 & 24,219 & 0.3 & 42 & 1153 & 3.6 & 938 & 111,455 & 0.8 \\
\hline
\end{tabular}

2. Space-time model results

The $95 \% \mathrm{CR}$ for the temporal random effect included the number 1 in all the years, indicating that in Spain as a whole, there were no significant changes between consecutive years in the risk of becoming infected or newly infected. Even though at country level there was no significant temporal trend, at county level bTB temporal 
evolution was more evident and spatially heterogeneous. For some years, some counties presented a significant increase, or decrease, in the risk of being infected or newly infected, compared to the previous year (Figures 3 and 4, respectively).

The final models for prevalence and incidence included 2 and 3 covariates respectively (Table 3). Movements from counties with high incidence $(>1 \%)$ and presence of bullfighting cattle herds had a positive relation with both the risk of being positive and the risk of being new positive. Specifically, we found that herds located in counties with the highest number of risk movements (categories 3 and 4) had a risk of becoming positive or new positive between 1.5 and 1.7 times higher compared to those counties with the lowest number of risk movements (category 1). Moreover, counties with presence of bullfighting herds had a risk between 1.3 and 1.5 higher of becoming positive or new positive as compared with counties with absence of bullfighting herds. On the other hand, the abundance of red deer improved the specification of the model for new infections, explaining part of the risk heterogeneity, but the relationship was not statistically significant.

Table 3. Covariates included in the final model selected according to the deviance information criterion, statistical coefficients and their standard deviations (SD) and 95\% credible intervals. Coefficients are relative to the lowest values (categories 1). Significant categories are marked with an $(*)$.

\begin{tabular}{llccccc}
\hline Model & Covariate & Category & Coefficient & SD & $\mathbf{2 . 5 \%}$ & $\mathbf{9 7 . 5 \%}$ \\
\hline Space-time prevalence & High risk movements & 2 & 1.07 & 1.16 & 0.79 & 1.46 \\
& & $3^{*}$ & 1.71 & 1.17 & 1.25 & 2.33 \\
& & $4^{*}$ & 1.66 & 1.18 & 1.19 & 2.33 \\
\cline { 2 - 7 } & & $2^{*}$ & 1.56 & 1.14 & 1.19 & 2.05 \\
\hline
\end{tabular}




\begin{tabular}{lllllll}
\hline Space-time incidence & High risk movements & 2 & 1.03 & 1.15 & 0.78 & 1.38 \\
& & $3^{*}$ & 1.67 & 1.15 & 1.25 & 2.23 \\
& $4^{*}$ & 1.58 & 1.17 & 1.16 & 2.16 \\
\cline { 2 - 6 } & Bullfighting & $2^{*}$ & 1.33 & 1.13 & 1.05 & 1.70 \\
\hline Red deer abundance & 2 & 0.92 & 1.14 & 0.71 & 1.20 \\
& 3 & 1.11 & 1.15 & 0.84 & 1.46 \\
& 4 & 0.95 & 1.18 & 0.69 & 1.32 \\
\hline
\end{tabular}

The relative risks of the prevalence (RRP) and incidence (RRI) in Spain, throughout the years of study, are represented in Figures 5 and 6 . The results of the model showed that the RRP and RRI were higher in counties located in central and southern Spain, throughout the whole period of study.

\section{Discussion}

According to a previous study, the risk of being positive or new positive, at county level, was not homogeneous throughout Spain, being higher in the central and southern areas of the country (Allepuz et al., 2011). However, in that study the temporal and space-time temporal trends could not be evaluated as just spatial models were used. Our results show that, the temporal trend at country level was not statistically significant for any of the years of study. The result of the temporal random effect of our models has to be interpreted based on its specification (i.e., autoregressive of order 1). In this sense, this random effect indicates the increase or decrease in the probability of disease compared with the previous year, but not as the temporal trend for the whole period of study. However, the temporal trend in herd prevalence from 2006 to 2011 has been reported to significantly decrease, from $1.8 \%$ in 2006 to $1.3 \%$ in 2011 (Anon., 2012b). 
Therefore, both results are not necessarily contradictory, as there may be no significant improvements in the bTB eradication campaign between consecutive years, but a significant temporal trend for the whole period.

At county level, the evolution has been more heterogeneous, as some counties presented significant space-time interactions. This result indicates an increase or a decrease on the risk in that area in that specific year, and it is related with the presence of short-latency underlying factors (i.e. not occurring in a regular manner over time) (Abellan et al., 2008). It is difficult to have reliable information about which shortlatency uncontrolled factors could be responsible for those significant space-time interactions, but we speculate that factors such as changes in the personnel in charge of the implementation of the diagnostic tests, intensification of controls, or the application of immediate depopulation, could be related to them.

Our results indicate that movements of animals from counties with high incidence $(>1 \%)$ were positively correlated with the risk of being positive and new positive. This is in agreement with studies conducted in the United Kingdom, where movements of animals were identified as a significant risk factor for bTB (Gilbert et al., 2005; Gopal et al., 2006; Green et al., 2008). When animal movements take place, different situations may occur that expose the animals to new infections. In our data base it exist different types of movements: to shared grasslands, livestock markets and other farms. Pre and post-movement controls should be realized (Anon., 2012a) although problems with the sensitivity of IDT test (de la Rua-Domenech, et al., 2006) may originate the existence of infected animals in the herd which could spread the disease to other farms after the movement. Also the existence of anergic animals and the possible contact with cattle from other farms, other domestic animals or even with wildlife reservoirs may explain the animal movement as a risk factor. 
The results indicate that the risk of a herd being infected or newly infected was higher in counties which had bullfighting herds. The type of production, in particular fighting bulls, has been described as potential risk factor for bTB infection in Spain by numerous authors (Rodriguez-Prieto et al., 2012; Anon, 2012b; Allepuz et al., 2011; Boadella et al., 2011). Fighting bulls are difficult to handle because of their vigor and the fact that they are managed in extensive areas with difficult access, which implies that some of the animals of the herd may not be tested (Aranaz et al., 2006; RodríguezPrieto et al., 2012). Moreover, bullfighting herds were introduced gradually into the eradication program context since 2004 (RD 1939/2004). These reasons may explain why the presence of bullfighting herds increases the risk of a herd becoming infected or newly infected. In fact, to tackle this problem, a new legislation (RD 186/2011), which reinforces the controls in bullfighting herds, has been introduced in 2011.

Our results show a positive correlation, although not significant, between the red deer abundance and the risk of new infections. The possible role of wild animals, mainly wild boar and red deer, as reservoirs of bTB in Spain, has been suggested in different studies (Rodriguez-Prieto et al., 2012; Gortázar et al. 2011; Rodriguez 2010; Naranjo et al., 2008; Vicente et al., 2006; Aranaz et al. 2004). In central and southern Spain, high prevalences are reported in these species, particularly in areas of high density (e.g. Gortázar et al., 2008; Castillo et al., 2011; Acevedo et al., 2007, 2008; Boadella et al., 2011; García-Bocanegra et al., 2012). Molecular typing has shown that different wildlife species such as red deer, fallow deer (Dama dama) and wild boar are infected with the same $M$. bovis spoligotypes as cattle, and that they may be maintained in the same area over time even in the absence of contact with domestic ruminants (Aranaz et al., 1996, 2004; Gortazar et al., 2005; Parra et al., 2005; Hermoso de Mendoza et al., 2006; Romero et al., 2008; Naranjo et al., 2008; Rodríguez et al., 2009). 
As only data for red deer abundance was available for the whole Spain, this was the species whose role was assessed. However, as wild boar and red deer have similar ecological requirements (Acevedo et al., 2011), their distributions are highly correlated, and therefore red deer abundance may be considered as a proxy measure of wild host abundance.

Even though there is no official data on the prevalence of caprine tuberculosis, the disease is considered to be endemic in Spain (Liébana et al., 1998), and therefore the presence of goats has also been suggested as a potential risk for bTB (Rodriguez et al., 2011; Álvarez et al., 2007). In a recent study, it was evidenced that goat herds infected with tuberculosis may pose a threat to neighboring bovine herds (Napp et al., 2013). Nevertheless, our results did not show any significant correlation between number of goat herds or mixed cattle-goat farms and the risk of a herd being infected or newly infected.

In the interpretation of the covariates included in the space-time model, it is important to keep in mind the spatial scale at which the analysis was done, as the relationship between the variables may change with the selection of different areal units (Meliker et al., 2011). While these kinds of models are useful to identify broad-scale spatio-temporal trends, they hide farm level heterogeneity, a phenomenon known as the ecological fallacy. Therefore, relations found at the aggregated level do not have to be the same at the individual level, and therefore, caution has to be taken when drawing conclusions from disease data summarized at the area level and should be corroborated by analysis at local scale.

The prevalence and the incidence of disease were the parameters used to evaluate the risk of bTB infection instead of the standardized mortality/morbidity ratio (SMR). SMR describes the odds of being in the disease group rather than the background group 
(Lawson et al., 2003), by calculating the ratio between the observed number of cases and the number that would have been expected in a standard population (Pfeiffer et al., 2008). It is very useful for disease mapping of aggregated (e.g. county) data (Pfeiffer et al., 2008), and it was previously used to describe the risk of bTB in Spain (Allepuz et al., 2011). However, as we developed a space-time model, aimed at comparing the risk of bTB from one year to the previous year, a ratio between prevalences or incidences seemed easier to interpret than a ratio between SMRs.

Regarding, the application of space-time models, there are a variety of formulations that have been proposed for the spatiotemporal analysis of the pattern of the risk of a disease, and it is not totally clear which one would be the most useful (Lawson et al., 2009). Different approaches, such as the use of mixed models (Held et al., 2005; Paul et al., 2008), different specifications for the random temporal and space-time temporal components (Bernardinelli et al., 1995; Knorr-Held., 2000; Abellan et al., 2008; Martínez-Beneito et al., 2008) and the use of non-separable space-time interactions (Knorr-Held., 2000), have been proposed. In the present work we used one of the formulations proposed in Knorr-Held (2000). By this formulation, the correlated and uncorrelated spatial components are defined as constant in time, and there are separate temporal and space-time interaction terms. Within this specification an autoregressive prior distribution allowing a non-parametric temporal and space-time trend was used. We did not formally compare the performance of different space-time models specifications, as it was beyond the scope of this study. The model used has been reported to give a parsimonious representation of the space-time behavior in risk (Knorr-Held, 2000; Lawson et al., 2009), and therefore we believe it gives an accurate representation of the variability of bTB across Spain during the study period. 
Space-time models are usually solved by the use of Markov chain Monte Carlo (MCMC) algorithms. However, they are computationally expensive and may induce large errors in parameter estimates (Schrodle et al., 2011). An alternative method to compute the parameters of interest, is the integrated nested Laplace approximations (INLA), recently proposed by Rue et al. (2009). The major advantage of this method is that it is computationally much faster than MCMC, returning precise parameter estimates. The application of space-time models using INLA is getting more common and, among others, it has been used to assess the space-time evolution of bovine viral diarrhea eradication in Switzerland (Schrödle 2011), to predict the areas with the highest potential for West Nile Virus introduction and amplification in Italy (Bisanzio et al., 2010), and to estimate air quality also in Italy (Cameletti et al., 2012).

\section{\# References}

Abellan, J.J., Richardson, S., Best, N., 2008. Use of space-time models to investigate the stability of patterns of disease. Environ. Health Perspect. 116, 1111-1119.

Acevedo, P., Vicente, J. Höfle, U., Cassinel, J., Ruiz-fons, F., Gortazar, C., 2007. Estimation of European wild boar relative abundance and aggregation: a novel method in epidemiological risk assessment. Epidemiol. Infect. 135, 519-527.

Acevedo P, Ruiz-Fons F, Vicente J, Reyes-García AR, Alzaga V, Gortázar C (2008) Estimating red deer abundance in a wide range of management situations in Mediterranean habitats. Journal of Zoology 276: 37-47.

Acevedo, P., Ruiz-Fons, F., Estrada, R., Márquez, AL., Miranda, MA., Gortázar, C., Lucientes, J. 2010. A Broad Assessment of Factors Determining Culicoides imicola Abundance: Modelling the Present and Forecasting Its Future in Climate Change Scenarios. PLoS ONE 5(12): e14236. doi:10.1371/journal.pone.0014236

Acevedo, P., Farfán, M., Márquez, A., Delibes-Mateos, M., Real, R., Vargas, J., 2011. Past, present and future of wild ungulates in relation to changes in land use. Landscape Ecology, Vol. 26, Num. 1, 19-31(13).

Allepuz, A., Casal, J., Napp, S., Saez, M., Alba, A., Vilar, M., Domingo, M., Gonzalez, M.A., Duran-Ferrer, M., Vicente, J., Alvarez, J., Munoz, M., Saez, J.L., 2011. Analysis 
of the spatial variation of bovine tuberculosis disease risk in spain (2006-2009). Prev. Vet. Med. 100, 44-52.

Anon., 2012a. Programa Nacional de Erradicación de Tuberculosis Bovina presentado por España para el año 2012(http://rasve.mapa.es//Publica/Programas/).

Anon., 2012b. Informe final técnico-financiero, programa nacional de la tuberculosis bovina, año 2011 (http://rasve.mapa.es//Publica/Programas/).

Aranaz A, de Juan L, Montero N, Sánchez C, Galka M, Delso C, Álvarez J, Romero B, Bezos J, Vela AI, Briones V, Mateos A, Domínguez L: Bovine tuberculosis (Mycobacterium bovis) in wildlife in Spain. J Clin Microbiol 2004, 42:2602-2608.

Aranaz, A., Liébana, E., Mateos, A., Dominguez, L., Vidal, D., Domingo,. M., Gonzolez, O., Rodriguez-Ferri, E.F., Bunschoten, A.E., Van Embden, D.A., Cousins, D., 1996. Spacer oligonucleotide typing of Mycobacterium bovis strains from cattle and other animals: a tool for studying epidemiology of tuberculosis. Journal of Clinical Microbiology. p. 2734-2740.

\section{Methodologic Issues and Approaches to Spatial Epidemiology}

Linda Beale,1 Juan Jose Abellan,1,2 Susan Hodgson,3 and Lars Jarup1

1Small Area Health Statistics Unit, Department of Epidemiology and Public Health, Imperial College London, London, United Kingdom;

2CIBER Epidemiología y Salud Pública (CIBERESP), Spain; зInstitute of Health and Society, Newcastle University, Newcastle Upon Tyne,

United Kingdom 2008

Besag, J., York,J., Mollie,A., 1991. Bayesian image restoration with applications in spatial statistics. Ann.Inst.Math.Stat. 43,1-20.

Best N., Richardson S., Thomson A. (2005). A comparison of Bayesian spatial models for disease mapping. Stat. Methods in Medical Research 14, 35-39.

Bisanzio, D., Giacobini, M., Bertolotti, L., Mosca, A., Balbo, L., Kitron, U., VazquezProkopec, G.M. 2011. Spatio-temporal patterns of distribution of West Nile virus vectors in eastern Piedmont region, Italy. Parasites \& Vectors, 4:230.

Boadella M, Gortazar C, Acevedo P, Carta T, Martín-Hernando MP, de la Fuente J, Vicente J: Six recommendations for improving monitoring of diseases shared with wildlife: examples regarding mycobacterial infections in Spain. Eur J Wildl Res 2011, 57:697-706.

Cameletti M., Lindgren F., Simpson D., Rue H., 2012. Spatio-temporal modeling of particulate matter concentration through the SPDE approach. ASta, 2012.

Castillo, L., Fernández-Llario, P., Mateos, C., Carranza, J., Benítez-Medina, J.M., García-Jiménez, W., Bermejo-Martín, F., Hermoso de Mendoza, J. 2011. Management practices and their association with Mycobacterium tuberculosis complex prevalence in red deer populations in Southwestern Spain. Prev. Vet. Res. 98, 58-63.

de la Rua-Domenech, R., Goodchild, A.T., Vordermeier, H.M., Hewinson, R.G., Christiansen, K.H., Clifton-Hadley, R.S., 2006. Ante mortem diagnosis of tuberculosis 
in cattle: A review of the tuberculin tests, gamma-interferon assay and other ancillary diagnostic techniques. Res. Vet. Sci. 81, 190-210.

Gilbert, M., Mitchell, A., Bourn, D., Mawdsley, R., Clifton-Hadley, R., Wint, W. 2005. Cattle movements and bovine tuberculosis in Great Britain. Nature letters. Vol 435/26.

Gortázar C, Delahay RJ, McDonald RA, Boadella M, Wilson GJ, Gavier-Widen D, Acevedo P (2012) The status of tuberculosis in European wild mammals. Mammal Review 42: 193-206

C. Gortázar, M.J. Torres, P. Acevedo, J. Aznar, J.J. Negro, J. de la Fuente \& J. Vicente (2011) Finetuning the space, time, and host distribution of mycobacteria in wildlife. BMC Microbiology 11, 27

Gortázar C, Torres J, Vicente J, Acevedo P, Reglero M, de la Fuente J, Negro JJ, AznarMartín J: Bovine tuberculosis in Doñana Biosphere Reserve: the role of wild ungulates as disease reservoirs in the last Iberian lynx strongholds. PLoS One 2008, 3:e2776.

Gortázar C, Acevedo P, Ruiz-Fons F, Vicente J: Disease risks and overabundance of game species. Eur J Wildl Res 2006, 52:81-87.

Gortázar C, Vicente J, Samper S, Garrido JM, Fernández-de-Mera IG, Gavín P, Juste RA, Martín C, Acevedo P, de La Puente M, Höfle U: Molecular characterization of Mycobacterium tuberculosis complex isolates from wild ungulates in south-central Spain. Vet Res 2005, 36:43-52.

Held L, Höhle M, Hoffmann MA. (2005). A statistical framework for the analysis of multivariate infectious disease surveillance counts. Statistical Modelling 5, 187-205.

Held, L; Schrödle, B; Rue, H (2010). Posterior and Cross-validatory Predictive Checks: A Comparison of MCMC and INLA. In: Kneib, T; Tutz, G. Statistical Modelling and Regression Structures - Festschrift in Honour of Ludwig Fahrmeir. Berlin, 91-110. ISBN 978-3-7908-2412-4.

Humblet, M.F., Boschiroli, M.L., Saegerman. C., 2009. Classification of worldwide bovine tuberculosis risk factors in cattle: a stratified approach. Vet. Res. 40:50.

Knorr-Held, L., 2000. Bayesian modelling of inseparable space-time variation in disease risk. Stat. Med. 19, 2555-2567.

Krajewska, M., Kozinska, M., Zwolska, Z., Lipiec, M., Augustynowicz-Kopec, E., Szulowski, K., 2012. Human as a source of tuberculosis for cattle. first evidence of transmission in poland. Vet. Microbiol. 159, 269-271.

Liébana, E., Aranaz, A., Urquía, J.J., Mateos, A., Domínguez, L. 1998. Evaluation of the gamma-interferon assay for eradication of tuberculosis in a goat herd. Aust. Vet. J. Vol 76, 1. 
Lindgren F., Rue H., Lindstrom J., 2011. An explicit link between Gaussian fields and Gaussian Markov random fields: The SPDE approach (with discussion). Journal of the Royal Statistical Society, Series B, 73 (4):423-498, 2011.

Mark S. Handcock, David R. Hunter, Carter T. Butts, Steven M. Goodreau, and Martina Morris (2003). statnet: Software tools for the Statistical Modeling of Network Data. URL http://statnetproject.org

Martínez-Beneito et al., (2008). "An autoregressive approach to spatio-temporal disease mapping."

Paul M., Held L., Toschke A. M. (2008). Multivariate modelling of infectious disease surveillance data. Statist. Med.; 27:6250-6267

Meliker, J.R., Sloan, C.D., 2011. Spatio-temporal epidemiology: Principles and opportunities. Spat Spatiotemporal Epidemiol. 2, 1-9.

Naranjo V, Gortázar C, Vicente J, de la Fuente J: Evidence of the role of European wild boar as a reservoir of tuberculosis due to Mycobacterium tuberculosis complex. Vet Microbiol 2008, 127:1-9.

Phillips, C.J.C., Foster, C.R.W., Morris, P.A., Teverson, R., 2004. The transmission of Mycobacterium bovis infection to cattle. Res. Vet. Science 74, 1-15.

Quantum GIS Development Team, 2012. Quantum GIS Geographic Information System. Open Source Geospatial Foundation Foundation. Available: http://qgis. osgeo.org.

R Core Team. A Language and Environment for Statistical Computing, R Foundation for Statistical Computing. Vienna, Austria, 2012. ISBN 3-900051-07-0, http://www.Rproject.org.

Rodríguez, E., Sánchez, L. P., Pérez, S., Herrera L., Jiménez, M. S., Samper, S., Iglesias, M. J., 2009. Human tuberculosis due to Mycobacterium bovis and M. caprae in Spain, 2004-2007. Int J Tuberculosis Dis 13(12):1536-1541.

Rodríguez-Prieto, V., Martínez-López, B., Barasona, J.A., Acevedo, P., Romero, B., Rodriguez-Cmapos, Sabrina., Gortázar, C., Sánchez-Vizcaíno, J.M., Vicente, J., 2012. A Bayesian approach to study the risk variables for tuberculosis occurrence in domestic and wild ungulates in South Central Spain.\# faltan las páginas

Romero B, Aranaz A, Sandoval A, Álvarez J, de Juan L, Bezos J, Sánchez C, Galka M, Fernández P, Mateos A, Domínguez L: Persistence and molecular evolution of Mycobacterium bovis population from cattle and wildlife in Doñana National Park revealed by genotype variation. Vet Microbiol 2008, 132:87-95.

Rue, H., Martino, S., Chopin, N., 2009. Approximate Bayesian inference for latent Gaussian models using inte-grated nested Laplace approximations (with discussion). Journal of the Royal Statistical Society, Series B, 71(2):319\{392, 2009. 
Simpson, D., Illian, J., Lindgren, F., Sørbye, S.H., Rue, H., 2011. Going off grid:

Computationally efficient inference for log-Gaussian Cox processes. NTNU Technical report 10/2011.

Schrödle B., Held L., 2010. Spatio-temporal disease mapping using INLA.

Environmetrics. DOI: 10.1002/env.1065.

Spiegelhalter DJ, Thomas A, Best NG, Lunn D: WinBUGS Version 1.4 Users Manual 2003. Cambridge: MRC Biostatistics Unit; 2011. http://www.mrc-bsu.cam.ac.uk/bugs Accessed 2 December.

Torgerson, P.R., Torgerson, D.J., 2010. Public health and bovine tuberculosis: What's all the fuss about? Trends Microbiol. 18, 67-72.

Vicente J, Höfle U, Garrido JM, Fernández-de-Mera IG, Juste R, Barral M, Gortazar C: Wild boar and red deer display high prevalences of tuberculosis-like lesions in Spain. Vet Res 2006, 37:107-119.

Zuur, A.F., Ieno, E.N., Walker, N.J., Saveliev, A.A., Smith, G.M., 2009. Mixed Effects Models and Extensions in Ecology with R. Springer Science + Business Media,

LLC, New York. 